\title{
Otros-maices: trayectorias y transformaciones culinarias del maíz en Ambato (Catamarca, Noroeste Argentino)
}

\author{
Francisco PaZzarelLi \\ Instituto de Antropología de Córdoba, CONICET-Universidad Nacional de Córdoba (Argentina) \\ fpazzarelli@hotmail.com
}

Recibido: 26 de septiembre de 2012

Aceptado: 30 de junio de 2013

\begin{abstract}
RESUMEN
Este trabajo describe y analiza las trayectorias y transformaciones culinarias del maíz en contextos domésticos de Ambato (Catamarca, Noroeste Argentino), durante los siglos VI-XI, a partir del estudio de distintos tipos de evidencias arqueológicas. Los modos en que las trayectorias y transformaciones se implicaban en las dinámicas cotidianas nos permiten sugerir, a tono con otros planteamientos realizados para el área andina, que la vinculación de este recurso a distintas dimensiones de la vida social residía, al menos en parte, en la posibilidad de verse involucrado en una diversidad de manipulaciones culinarias. Aquí argumentamos que estas manipulaciones, lejos de operar como mediaciones entre un estado «natural» del recurso y su ingesta, constituían instancias irreemplazables para la socialización del mismo, con objeto de producirlo como un otromaíz que entonces podía articular y ser articulado en diferentes relaciones de comensalidad; en este sentido, la ejecución de técnicas en la cocina suponía un proceso de «especificación culinaria».
\end{abstract}

Palabras clave: Maíz, trayectorias culinarias, transformaciones culinarias, especificación, Ambato.

\section{Other-Maizes: Paths and Culinary Transformation of Maize in Ambato (Catamarca, Northwestern Argentina)}

\begin{abstract}
This paper describes and analyzes the paths and culinary transformations of maize in domestic contexts at Ambato (Catamarca, northwest Argentina), during $6^{\text {th }}-11^{\text {th }}$ centuries, from the study of different types of archaeological remains. The ways in which the trajectories and transformations involved in daily dynamics allow us to suggest, in line with another works for the Andean region, that the linking of this resource to different dimensions of social life was, at least in part, because of its involvement in a variety of culinary manipulations. These manipulations, far from operating as mediations between a «natural» state of the resource and its intake, has to be seen as irreplaceable instances of socialization of it, in order to produce it as an other-maize that could then articulate different relations of commensality; the implementation of techniques in the kitchen, then, involved a process of «culinary specification».
\end{abstract}

Key words: Maize, culinary paths, culinary transformations, specification, Ambato.

Sumario: 1. Introducción. 2. El maíz y sus transformaciones en Ambato. 3. Discusión: trayectorias, transformaciones, especificaciones. 4. Conclusiones. 5. Referencias bibliográficas.

\section{Introducción}

La importancia del maíz en la vida económica y ritual de la región andina ha sido frecuentemente argumentada mediante diferentes aproximaciones que han permitido comprender cómo este recurso se imbricaba en distintas dimensiones de la vida social de los grupos indígenas-campesinos (Murra 1973; Ossio 1988; Randall 1993). Muchos acercamientos se caracterizaron por centrarse en las formas en que el maíz era producido, distribuido y consumido en ocasiones «domésticas» y «rituales», ex- 
plorando los modos en que todo ello se inscribía en contextos sociales específicos. Pero fueron los análisis vinculados con su ingesta, especialmente, los que permitieron argüir en torno a la capacidad del maíz para vincularse y habilitar distintas relaciones de comensalidad: así lo atestiguaría su presencia constante en las dietas cotidianas (Contreras Hernández 1985; Paredes Candia 1990; Vokral 1991; Weismantel 1994), como bebida fermentada (chicha) en fiestas y celebraciones (Randall 1993; Allen 2002; Bray 2003; Abercrombie 2006) o como «ofrenda» en distintas situaciones rituales (Murra 1973; van den Berg 1989; Fernández Juárez 1997; Abercrombie 2006). Muchos de estos supuestos, construidos desde la etnohistoria y la etnografía, también fueron discutidos desde la arqueología (Hastorf y Johannessen 1993; Bray 2003; Goldstein 2003; Nielsen 2010), permitiendo dotar de profundidad histórica a las evidencias de consumo de maíz en sus distintas formas.

Generalmente, estas miradas se vinculan, de diferente modo, a consideraciones compartidas acerca de los ritos de comensalidad en los Andes, que sugieren la relevancia del consumo de ciertos alimentos para producir y reproducir relaciones sociales de pertenencia, afinidad o parentesco, sea entre agentes humanos o no humanos (Spedding 1993; Fernández Juárez 1997; Weismantel 1994, 1998; Nielsen 2010). Sin embargo, creemos que un análisis que pretenda comprender cómo determinadas comidas se inscriben en relaciones sociales locales no puede desestimar el espacio social de la cocina, donde se ejecutan las transformaciones culinarias que habilitan la existencia de aquellos platos que luego se consumen.

En este sentido, aquí argumentamos ${ }^{1}$ que, mediante su inscripción en las relaciones de transformación específicas de una cocina, el maíz es colocado en un plano de contingencia diferente del que ocupaba en los campos o en los almacenes, reproduciéndose como algo distinto y adquiriendo una nueva forma (un nuevo «cuerpo») que habilita, entonces, diferentes relaciones de comensalidad y socialidad. En otras palabras, esto nos llevará a preguntarnos sobre las posibles relaciones entre las transformaciones culinarias, que permiten producir las texturas y sabores deseados, y aquellos eventos de comensalidad auspiciados por la posibilidad de ingesta de ciertos platos.

Este supuesto conlleva dos consecuencias analíticas: por un lado, coloca a la cocina como un locus de transformación específico que habilita la existencia de aquellos espacios de comensalidad que parecían sólo auspiciados por la presencia de un recurso o comida; por otro lado, considera que las transformaciones culinarias no transforman recursos ya existentes, sino que los producen al re-colocarlos en un nuevo plano de contingencia. En este sentido y vinculadas al problema particular sobre el que discurriremos aquí, estas consideraciones permitirían sugerir que la mentada «versatilidad» del maíz en los Andes se funda en la manipulación de las transformaciones a las que es sometido y a partir de las cuales es re-producido como algo nuevo, como un otro-maíz que porta nuevas texturas, temperaturas, densidades y formas. Es así que las cocinas podrían ser pensadas como espacios donde se propicia un proceso de «especificación culinaria».

\footnotetext{
1 Este artículo presenta algunos de los argumentos de la tesis doctoral, defendida en 2012 en el Doctorado en Ciencias Antropológicas de la Universidad Nacional de Córdoba, Argentina (Pazzarelli 2012a).
} 
La idea de «especificación» ha sido trabajada por distintos autores en el área amazónica (Viveiros de Castro 1986; Vilaça 2002) con objeto de analizar los modos en que se producen cuerpos emparentados. Tal como afirma Vilaça (2002), un proceso como éste supone la ejecución de técnicas que permiten especificar (y moldear) la «naturaleza» de los cuerpos recién nacidos, recortándolos de la matriz de «socialidad» general en la que se inscriben para producir un «humano» que pueda ser transformado en «pariente». En otras palabras, se trata de un proceso, ejecutado a partir de técnicas particulares (como la alimentación, el ingreso paulatino en las actividades domésticas, el cuidado personal) que permite, antes que una «culturalización», una «especificación» de la «naturaleza» de aquellos cuerpos que, de otra manera (y mediante otras técnicas), podrían ser transformados en parientes de otros (de algunos animales, por ejemplo). Estos planteamientos no sólo permiten poner en cuestión ciertos aspectos clásicos de la teoría del parentesco (para un ejemplo similar de los Andes ecuatorianos, ver Weismantel 1994, 1998), sino que argumentan a favor de la vinculación entre la forma y la materialidad (en este caso, de los cuerpos) y las capacidades agentivas de esos sujetos. En otras palabras: en la manipulación de los cuerpos (de las formas físicas y de las materialidades) se definen las capacidades agentivas particulares de un ser.

¿Cuáles son las consecuencias de una apelación teórica de este tipo? Una categoría como la de «especificación» brinda herramientas analíticas para colaborar en la comprensión de las técnicas culinarias al margen de los esquemas teleológicos, guiados por el consumo, en los que generalmente se inscriben. A nuestro entender, las transformación culinarias deberían ser comprendidas como operadores lógicos que permiten una operación de distinción, a partir de las cuales el maíz cosechado adopta diferentes tipos de formas físicas y materialidades (o «cuerpos»), en virtud de las cuales se definirán sus capacidades agentivas. En otras palabras, sostenemos que el espacio social de las cocinas andinas opera como un locus que permite traer a la vida a nuevos maíces, «distintos» de aquellos que fueron cosechados y luego ingresados en los hogares, mediante una especificación de sus «cuerpos» (texturas, densidades, temperaturas, colores) lograda a partir de la ejecución de distintas técnicas culinarias. Estos otros-maíces, con cuerpos diferentes a los que se cosecharon, son los que podrán habilitar luego distintas relaciones de comensalidad, en virtud de la manipulación que se ha hecho de sus capacidades agentivas (amplificándolas o reduciéndolas; por ejemplo, cf. Lévi-Strauss 1968).

Un acercamiento de este tipo supone abordar de forma densa las transformaciones culinarias. Algunas ideas vinculadas con ésta fueron parcialmente propuestas por otros autores (Hastorf y Johannessen 1993; Randall 1993) y por nosotros para algunos casos arqueológicos (Laguens et al. 2007; Laguens y Pazzarelli 2011); en todas ellas se destila la importancia de comprender de manera ajustada cuáles son y cómo se ejecutan las técnicas de transformación para evaluar las maneras específicas en que los «recursos» son socializados. Con el objetivo de discutir estos supuestos, en este trabajo presentamos las diferentes trayectorias y transformaciones culinarias del maíz en los contextos domésticos de la cultura de la Aguada, que se desarrolló en Ambato (Catamarca, Noroeste Argentino -NOA-) durante los siglos VI a XI de nuestra era. Estos contextos constituyen una oportunidad para discutir cómo un análisis centrado 
en la cultura material puede echar luz sobre aspectos que en ocasiones son olvidados por la etnografía: los objetos y técnicas concretas que se ejecutan en la cocina (Pazzarelli 2010, 2012a). Finalmente, el recorrido por las trayectorias del maíz en Ambato pondrá en evidencia ciertos modos particulares de estructuración del espacio doméstico y de sus lógicas culinarias.

\section{El maíz y sus transformaciones en Ambato}

El valle de Ambato corresponde al sector septentrional del Valle Central de la provincia de Catamarca (NOA) y se encuentra recorrido por el río Los Puestos. Forma parte del Distrito Chaqueño Serrano de la Provincia Chaqueña (Dominio Chaqueño) y posee un clima continental cálido. Hacia el este y el noreste limita con la provincia biogeográfica de las yungas, mientras que hacia el oeste y el noroeste lo hace con el monte y la prepuna (Assandri et al. 1991) (Figura 1).

El desarrollo de la cultura de La Aguada en Ambato (entre los siglos VI y XI de nuestra era) se habría fundado, entre otras cosas, en una modificación de modos de vida anteriores, a partir del surgimiento de diferencias sociales marcadas que se habrían reproducido en el tiempo y que han sido investigadas atendiendo a diferentes dimensiones de la vida social: la gestión de espacios productivos, la distribución diferencial de recursos y la utilización de los espacios arquitectónicos, entre otras (Laguens 2006, 2007). Entre estas diferencias se encontrarían aquellas enmarcadas y reproducidas en grandes eventos colectivos de agregación social, con fines "rituales" y/o "políticos", llevados a cabo en sitios de una configuración espacial y arquitectónica más compleja. Estos eventos habrían propiciado, entre otras cosas, situaciones de comensalidad colectiva en donde la provisión y ofrecimiento de bebidas y comidas constituirían parte de los mecanismos que coadyuvaban a que ciertas diferencias se reprodujeran. Estos argumentos fueron propuestos, con matices y diferencias, por diferentes autores (Gordillo 2009; Laguens 2006, 2007; Pérez Gollán 1991, 1994), y en algunos puntos se encuentran cercanos a los esgrimidos para otras regiones del mundo andino.

Aunque no es objetivo de este artículo realizar una evaluación comparativa de estos argumentos (algo que desarrollamos en otro lugar, Pazzarelli 2012a), lo cierto es que ellos evidencian la importancia de los eventos de comensalidad (el compartir comidas y bebidas) en las explicaciones de lo que constituyó el origen y reproducción de la cultura de La Aguada en Ambato; en especial, cuando se vinculaba con el consumo de bebidas fermentadas a base de maíz (chicha). Fue en función de la relevancia otorgada a estos eventos que iniciamos una exploración sistemática de la producción de comidas en Ambato, atendiendo a las técnicas culinarias y a la estructuración relacional de los espacios destinados a tales actividades (Laguens et al. 2007; Pazzarelli 2011, 2012a, 2012b). Ello nos permitió observar una diversidad de prácticas culinarias vinculadas de manera recurrente a espacios específicos y cuyas trayectorias alteraban la materialidad de los recursos hasta casi transformarlos por completo. En virtud de su importancia en la dieta cotidiana y de su presencia recurrente en el registro arqueológico, las trayectorias del maíz nos aportaron una imagen bastante ajustada de 
sus diferentes devenires dentro de los sitios, lo que permitió generar preguntas e hipótesis en torno de los eventos de comensalidad que dichas trayectorias podrían haber propiciado. El inicio de estas trayectorias, no obstante, se encontraba en las terrazas de cultivo.

La producción de maíz en Ambato se encuentra evidenciada por extensos sistemas de terrazas agrícolas que se extienden por gran parte de las laderas, en algunas de las cuales se identificaron microfósiles (fitolitos) afines a Zea mays (Figueroa 2010). Aunque todavía no es claro si la producción contaba con el aporte de agua de riego (la envergadura y extensión del sistema de canales todavía no cuenta con la resolución necesaria para confirmar esto), es posible argumentar que una cosecha anual de secano podría haber sido suficiente para abastecer los requerimientos de los sitios residenciales durante todo el año (almacenamiento mediante) (Figueroa 2010) ${ }^{2}$. Las terrazas generalmente se vinculan con estructuras de corrales; esto, sumado a los altos niveles de fósforo y materia orgánica identificados en las primeras (que sugieren prácticas de abono, posiblemente con guano) y a los análisis isotópicos realizados sobre huesos de llamas (que sugieren que eran alimentadas con los rastrojos de las cosechas), permitieron

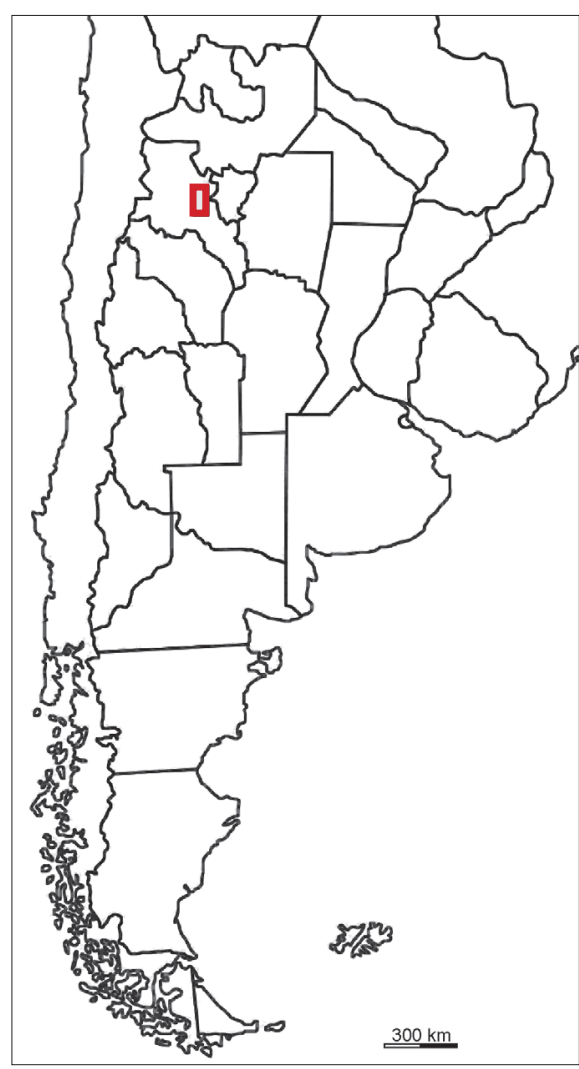

Figura 1: Ubicación del Valle de Ambato en el territorio actual de la Provincia de Catamarca, República Argentina. argumentar que la producción de maíz se encontraba íntimamente imbricada con los ciclos de la crianza de camélidos (Figueroa et al. 2010), tal como sucedía y sucede en otras regiones de los Andes. En el espacio definido por estas estructuras (terrazas y corrales) se encuentran también recintos y sitios habitacionales; sin embargo, la mayoría de ellos se encontraban emplazados en el fondo del valle, en ocasiones conformando agrupamientos a la manera de «aldeas» (Assandri 2007) que se confundían con el monte circundante, en donde el algarrobo (Prosopis sp.) constituía uno de los árboles característicos (entre muchos otros) y sus vainas eran frecuentemente consumidas, como veremos luego.

En estos espacios de vivienda, sobre los que nos centraremos aquí, se reconocen ciertos aspectos generales que caracterizan la organización espacial interna de todos ellos: están conformados por módulos rectangulares, en ocasiones adosados

\footnotetext{
2 Aún no existen evidencias concretas sobre la existencia de otros cultivos, pues los hallazgos realizados en contextos domésticos de macrorrestos y microrrestos afines a porotos (Phaseolus vulgaris) y de microrrestos vinculados a tubérculos y raíces no diferenciados, todavía no permiten distinguir el origen (silvestre o cultivado) de los mismos.
} 
entre sí, que pueden contar con espacios semiabiertos adyacentes, a la manera de galerías, que comunicaban con patios abiertos. Este conjunto de estructuras generalmente se encuentra contenido por muros perimetrales, fuera de los cuales podían existir montículos-basureros. Los módulos o habitaciones solían ser recintos cerrados y techados, que se comunicaban entre sí mediante vanos de acceso y que permitían acceder a las galerías mediante un escalón que sorteaba los $20 \mathrm{~cm}$ de diferencia entre un espacio y otro (los pisos de las habitaciones se encontraban entre los $70 \mathrm{~cm}$ y el metro de profundidad, mientras que los de las galerías y patios se hallaban unos 20 cm por encima). Las galerías eran espacios semitechados, abiertos hacia el sector que comunicaba con los patios, donde podían existir otros conjuntos de módulos y galerías similares. Esta configuración espacial se registra en la mayoría de los sitios, aunque el tamaño y la complejidad de su organización (cantidad de recintos, patios o galerías; distintos niveles de acceso) difiere: según algunos autores, los complejos residenciales de mayor tamaño dan cuenta de actividades de tipo extradoméstico vinculadas posiblemente a eventos de gran agregación social, como los ya mencionados, donde también se involucrarían los habitantes de los sitios más pequeños (Assandri 2007; Gordillo 2009). Los sitios se encuentran ubicados cronológicamente entre los siglos VI y XI de nuestra era, lo cual corresponde con el desarrollo de la cultura de La Aguada en la región; pero también existen otros sitios tempranos, generalmente montículos, ubicados entre los siglos I y V, previos a Aguada (para una discusión estratigráfica de estos sitios, ver Gastaldi 2012).

Los registros vinculados con el maíz en estos contextos son múltiples. Se ha recuperado como resto carbonizado (granos aislados y mazorcas) en los montículosbasurero, los cuales se constituían en los depósitos de «desechos» de actividades cotidianas (la cocina, entre ellas) de sitios monticulares tempranos («El Altillo»y «Martínez 3») y de otros que se ubican cronológicamente en el desarrollo de Aguada (algunos de gran complejidad espacial, como «Piedras Blancas» e «Iglesia de los Indios»). No obstante, si bien los registros son abundantes, las variedades identificadas sólo corresponden a Zea mays var. microsperma y var. Oryzaea (Gordillo 2009; Pazzarelli 2012a). Como microrresto, se identificaron gránulos de almidón afines a Zea mays en un molino, en manos de moler, en cuchillos de filita y en vasijas de distintos sitios residenciales. Como veremos, estos hallazgos pueden ser vinculados a las trayectorias y transformaciones por las que transitaba el maíz en sus diferentes formas; para avanzar sobre ello, a continuación describiremos el conjunto de técnicas en las que se involucraba, considerando la cultura material implicada y las diversas dinámicas cotidianas en las que estas prácticas podrían comprenderse.

Buena parte de los argumentos que siguen se fundan en la identificación de granos de almidón en artefactos. En muchos casos, el análisis de los daños existentes en la estructura de los gránulos permitió hipotetizar sobre los procesos tafonómicos (culinarios, en nuestro caso) que imprimieron huellas características e identificables sobre ellos (tal y como sugiere Babot 2003, 2004). Aquí exponemos un resumen de estos resultados, ya que la discusión respecto de las adscripciones taxonómicas y la identificación de daños fueron presentadas en otro trabajo (Pazzarelli 2012a); lo mismo se aplica al caso de la identificación y análisis de ácidos grasos y el análisis isotópico (Pazzarelli 2011, 2012a). 
Figura 2: a y b) Ejemplos de cuchillos de filita; en el primer caso, se observa una pátina que podría estar vinculada a su agarre y manipulación. c) Mano de molino típica; se observan restos adheridos a su superficie activa, donde se identificaron gránulos de almidón afines a Prosopis sp. d) Pequeña mano de mortero; se observan restos adheridos a su superficie activa, donde se identificaron gránulos de almidón afines a Zea mays y a Prosopis sp.
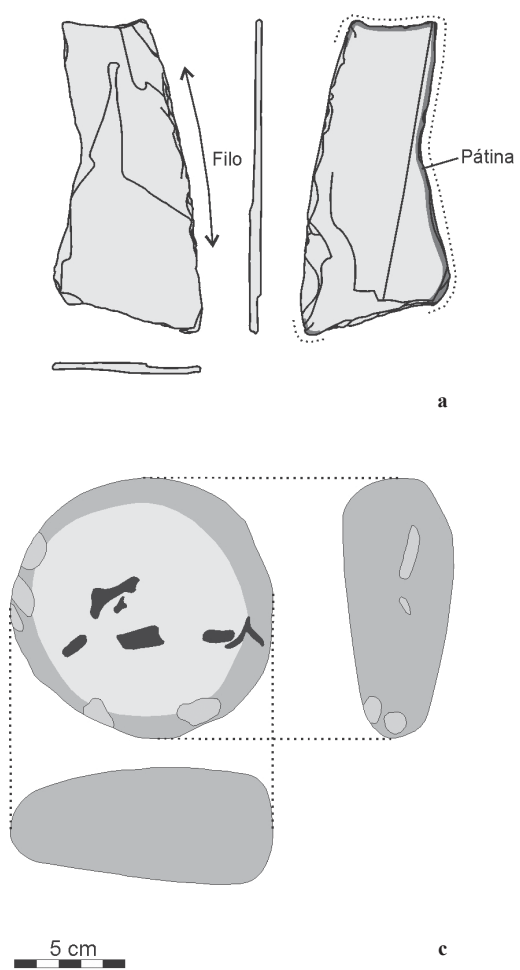
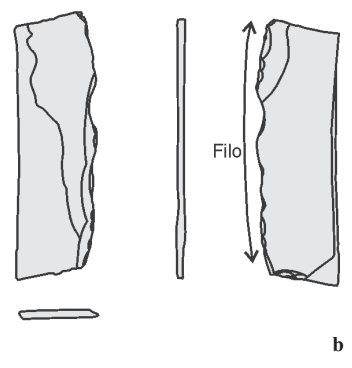

b

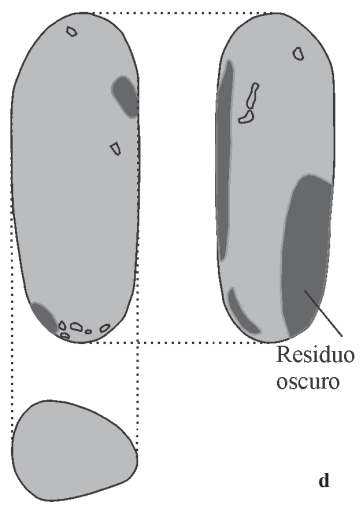

\subsection{Desgranado/rallado de choclos frescos}

El manejo de choclos frescos se formuló como hipótesis en virtud del hallazgo de gránulos de almidón afines a Zea mays sobre el filo de cuchillos de filita. Estos instrumentos (que varían entre los $66 \mathrm{~mm}$ y los $120 \mathrm{~mm}$ de alto, los 30mm y los 73 de ancho y los 1,5 y los 4,25 de espesor; Figura 2) se encuentran manufacturados sobre filitas locales, de estructura laminar planar, que forma lajas fácilmente. Generalmente, son instrumentos bifaciales, que presentan filos largos ubicados sobre uno de los laterales de la pieza y que se vinculaban al corte por presión y aserrado (Aschero 1975). Podían ser manejados con una mano y en algunos casos existen pátinas que podrían sugerir formas de agarre y manipulación (Figura 2a). En ocasiones se han hallado en conjuntos, en galerías de distintos sitios, ubicados bajo la línea de goteo de las mismas y cercanos a conjuntos de piedras de moler. También se los encuentra de forma aislada en el interior de los recintos y cercanos a los fogones utilizados para la cocción de alimentos.

La presencia de microrrestos afines al maíz sobre sus filos nos obliga a considerar su acción sobre choclos frescos (desgranado/rallado) o bien sobre comidas cocidas con base en el maíz. La primera hipótesis se funda en dos aspectos: por un lado, la fragilidad de la materia prima sobre la que se encuentran manufacturados los cuchillos dificultaría su acción sobre mazorcas secas, pues deberían ser constantemente afilados, en caso de que no se fracturaran rápidamente (considerando que, actualmente, para lograr el desgranado, las mazorcas secas deben ser golpeadas con un palo mientras están en el piso) (Figueroa 2010; Pazzarelli 2012a). Por otro lado, en estos cuchillos también se 
registraron gránulos de almidón afines a distintos tubérculos y raíces, lo que ha permitido proponer su uso en procesos de pelado y corte; es decir, en el manejo de productos frescos y blandos, algo que podría extenderse hacia la hipótesis del manejo del maíz como choclo. Si bien se trata de una posibilidad que debe seguir siendo indagada, es interesante, pues se trata de una técnica vinculada a los estados estacionales frescos $\mathrm{y} / \mathrm{o}$ blandos del maíz (y a cierto tipo de comidas) que habrían requerido del ejercicio de una fuerza moderada, a diferencia de los estados secos y duros de los granos deshidratados, que habrían sido de manejo y consumo recurrente, como veremos a continuación.

\subsection{Deshidratación y almacenamiento de mazorcas y granos}

Existen varios hallazgos que nos permiten pensar en los procesos de almacenamiento de los granos. Por un lado, contamos con la identificación de granos de almidón (en manos de moler y vasijas) que presentan daños en su estructura vinculados posiblemente con los efectos de la deshidratación (Babot 2003, 2004). Por otro lado, la alta frecuencia de gránulos registrada en artefactos de molienda permite sugerir que gran parte de la producción era deshidratada y luego procesada para lograr harinas; este secado de los granos pudo haber tenido lugar en cercanías de las terrazas de cultivo (donde existen algunas estructuras posiblemente vinculadas al almacenamiento temporal). Los granos luego deberían haber sido almacenados enteros, pues en caso de encontrarse pelados o partidos se pudrirían fácilmente; por esta misma razón, en contextos etnográficos no es posible conservar harinas por mucho tiempo y la molienda debe realizarse de forma cotidiana, como veremos luego (Babot 2004).

El registro de mayor envergadura vinculado al almacenamiento lo constituyen vasijas de gran tamaño (forma «A») que se ubicaban sobre todo en las galerías (a veces, ordenadas en filas, una junto a otra y ocupando espacios extensos), pero también en el interior de los recintos, muchas veces sostenidas por estructuras de piedra en el centro de las habitaciones. Según Gastaldi (2010), estas vasijas se caracterizan por ser restringidas, de contorno compuesto o inflexionado, con una sección inferior subglobular de base cónica (por lo que necesitaban de apoyo para mantenerse erguidas) y una superior subcilíndrica, con paredes convergentes o subtroncocónicas, con labio recto y un punto de inflexión que separa el cuello del cuerpo, sin asas. Poseen una porosidad que permitía la respiración de los recursos almacenados y una baja resistencia al shock térmico. Sus alturas oscilan entre los $253 \mathrm{~mm}$ y los $783 \mathrm{~mm}$; es decir, al tratarse de una clase de vasijas con dimensiones muy variadas, sus volúmenes también oscilan entre los 7,86 litros y los 261 litros, con un promedio de 76,57 litros (Gastaldi 2010). Se trata de los recipientes con mayores capacidades de contención en el valle. Muchas de ellas, además, presentan una decoración tricolor en su superficie externa y modelados con caras antropomorfas y antropozoomorfas (Figura 3). En una vasija de este tipo fueron hallados gránulos de almidón afines a Zea mays.

$\mathrm{Si}$ bien constituyen hallazgos frecuentes, estos recipientes no poseen una distribución homogénea (al menos en términos cuantitativos), ya que en aquellos sitios con una organización espacial más compleja se registra una mayor cantidad de recipientes (y, por tanto, de capacidades de almacenamiento) que en sitios pequeños. Y aunque otras vasijas (de otras formas y de tamaños menores) también podrían haber 

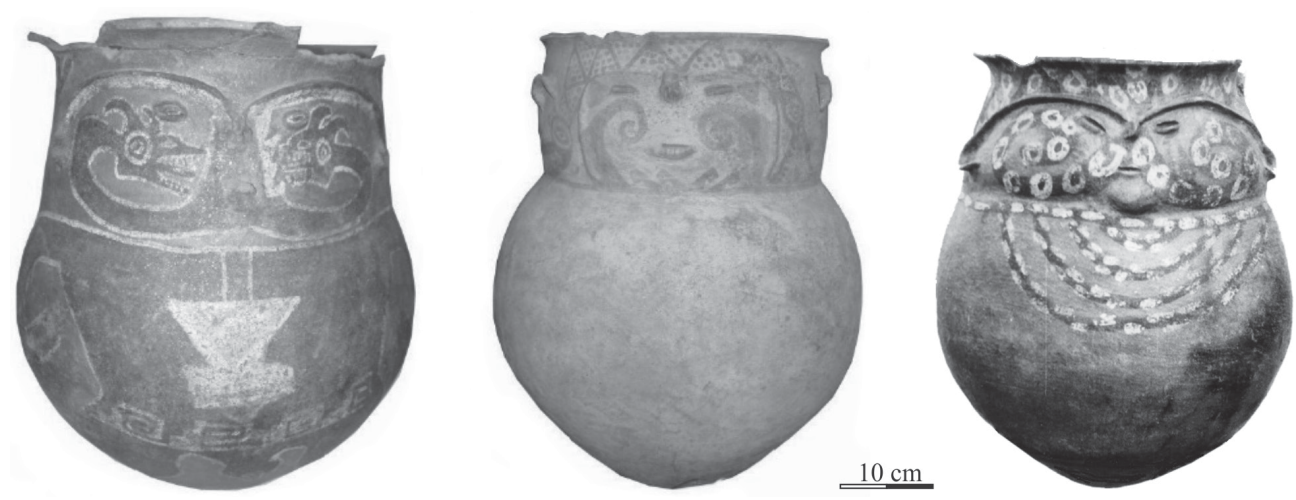

Figura 3: Vasijas de forma «A», destinadas al almacenamiento y a la fermentación.

servido para el almacenamiento de recursos (en menor volumen), los tamaños presentados por la clase «A» y su aparente inmovilidad en su ubicación en las galerías permite considerarlas como una de las expresiones materiales menos ambiguas en su vinculación con el almacenamiento de grandes volúmenes a largo plazo.

\subsection{Moliendas}

La molienda se encontraba definida por la ubicación de conjuntos de piedras de moler en los espacios semiabiertos de las galerías (es decir, donde se habría encontrado la mayoría de reservas de granos secos) y en el interior de los recintos, cercanos a los vanos de acceso; en algunos sitios también se registra el hallazgo aislado de manos de molino en los patios abiertos y junto a los fogones que se emplazaban en el interior de las habitaciones cerradas.

Los hallazgos más recurrentes corresponden a instrumentos activos, sobre todo de manos de molino (Figura 2c), con tamaños que permitían utilizar un solo brazo para su manejo y habilitaban una gran versatilidad en los movimientos posibles así como en el uso de bases de distinto tipo. En los pocos casos de manos que debían ser manejadas con ambos brazos, las curvaturas de sus superficies activas sugieren que debían ser utilizadas sobre bases planas, tal como las que presentan algunos de los molinos recuperados. Todas ellas poseen evidencias de desgastes pronunciados de las caras activas, lo que supone eventos de molienda intensivos y reiterados. La identificación de gránulos de almidón permitió comprobar que las manos de molino (de todos los tamaños) procesaban maíz (Zea mays), además de vainas de algarrobo (Prosopis sp.) y posiblemente tubérculos/raíces no diferenciados y porotos (Phaseolus sp). Las manos de mortero (Figura 2d) son de hallazgo mucho más ocasional y también se vinculaban con el procesamiento de maíz y vainas de algarrobo.

Los daños identificados en los gránulos de almidón recuperados en estos objetos se vincularían con los procesos de trituración, el posible calentamiento durante la misma (debido a la fricción entre la mano y la base, sobre todo en episodios de molienda intensiva) y la posible introducción de agua en la oquedad de los morteros para 
descascarar los granos (Babot 2003, 2004); en un caso, se identificaron gránulos con posibles evidencias de tostado, lo que podría sugerir procesos de cocción previos a la molienda, como veremos más adelante (Babot 2003; Pazzarelli 2012a).

Si tenemos en cuenta la mencionada dependencia respecto del maíz deshidratado y la imposibilidad de almacenar granos partidos o harinas por largos períodos de tiempo, podemos suponer que las actividades de molienda eran frecuentes y ocupaban buena parte de los días. Por otro lado, la alta frecuencia de manos de molino, que permitiría moler, triturar y pulverizar los granos (Babot 2004), sugiere que las harinas finas habrían sido de circulación frecuente, habilitando preparaciones y consumos específicos. Los morteros, de menor aparición, probablemente se vincularon con el descascarado, partido o primera trituración gruesa, instancias previas a la molienda fina. A diferencia de otros recursos, la presencia de maíz se registra en instrumentos de molienda de todo tipo y tamaño, lo que supone que los granos eran manejados con diferentes objetos y en distintos volúmenes. Sin embargo, la mayor cantidad de piedras de moler (incluyendo aquellas que debían ser manejadas con ambos brazos) se registran en sitios con una organización espacial y doméstica compleja, en donde también se identificaron los mayores volúmenes vinculados con el almacenamiento (Pazzarelli 2012a).

\subsection{Mezclas}

Si bien existen múltiples combinaciones posibles entre el maíz y otros recursos alimenticios para lograr diferentes preparaciones, aquí haremos referencia a dos de ellos, ambos comprobados mediante análisis de microfósiles. El primer caso se refiere a una pequeña mano de mortero que poseía un residuo oscuro, de aspecto harinoso, adherido a su superficie (Figura 2d); fue hallada junto a un fogón, en el interior de un recinto de un sitio pequeño («Martínez 1»). En el residuo se identificaron gránulos de almidón afines a Zea mays y a Prosopis sp., en lo que podría haber constituido una mezcla intencional de harinas. Este tipo de mezclas, que incluye recursos cultivados (maíz) y silvestres (vainas de algarrobo), es común en distintos grupos indígenascampesinos de las regiones chaqueñas y andinas, y forman parte de las «comidas secas» que se consumen de diferentes modos: el ulpo, por ejemplo, es una lejía compuesta por harina de algarroba y de maíz tostado que se consume directamente o diluida en agua o alguna infusión (Capparelli 2008) (un homólogo de otras harinas tostadas que se consumen en la región andina central); el patay y el bolanchao, por otro lado, son «panes» realizados a partir de la mezcla de diferentes harinas (entre las que se incluyen las citadas) que se amasan (aprovechando el jugo de los frutos silvestres molidos) y luego son prensadas o colocadas en moldes para ser cocidas al rescoldo de los fogones o al sol. Ambos productos pueden ser almacenados, transportados como reserva durante los viajes de intercambio o bien consumidos mientras se laborean los campos. En tres manos de moler de otro sitio («Piedras Blancas») se identificaron residuos pastosos idénticos (Figura 2) que permiten pensar que este tipo de preparaciones «secas» eran frecuentes (aunque en ellos sólo se recuperaron gránulos de almidón afines a Prosopis sp.).

El segundo tipo de mezcla es el que se producía en el interior de las ollas, probablemente durante el hervido de preparaciones semilíquidas. El análisis de dos resi- 
duos carbonizados del interior de vasijas destinadas a la cocción permitió identificar la presencia combinada de maíz y tubérculos y raíces no diferenciados. Si bien no es posible distinguir si estos residuos constituyen el producto de cocciones simultáneas o alternadas, es interesante considerar y seguir indagando especialmente la primera hipótesis, ya que se vincularía a las formas actuales de preparación de sopas y guisos.

En ambos casos (la mano de mortero y la vasija de cocción), los hallazgos se vinculan a los espacios interiores de los sitios, relacionados con objetos que se utilizaban junto o sobre los fogones (pequeña mano de mortero y vasija, respectivamente).

\subsection{Cocciones}

La cocción en Ambato se encuentra definida, casi exclusivamente, por la técnica del hervido en vasijas de distinto tipo (Gastaldi 2010; Pazzarelli 2012a). El hervido supone la cocción de los alimentos en un medio líquido que se lleva a ebullición. Permite, por un lado, incorporar a una olla con agua distintos tipos de recursos y en diferentes estados: molidos, cortados, picados y/o pelados; pero también habilita la incorporación de alimentos que ya han sido cocidos mediante otros métodos: tostados o freídos. En este proceso, los alimentos generalmente se incorporan uno a uno, dejando primero que el anterior hierva, lo que permite controlar los procesos de espesado, para lo cual también es imprescindible contar con un fuego regular y constante (Vokral 1991; Weismantel 1994). En Ambato, todos los fogones vinculados al manejo de recursos alimenticios se encuentran ubicados en el interior de las habitaciones, donde también suelen encontrarse recipientes vinculados con la cocción, instrumentos asociados al trabajo artesanal, restos óseos de diferente tipo y cuchillos de filita y manos de moler aisladas (Pazzarelli y Marconetto 2010). Veamos las vinculaciones entre el hervido sobre estos fogones y el manejo del maíz.

Las vasijas para las que existen evidencias de cocción de maíz eran de tipo restringidas subglobulares de cuello evertido, con un punto de inflexión que separa el cuello del cuerpo, con labio recto y redondeado, que pueden presentar asas horizontales o verticales remachadas. Poseen bases de formas cónicas y convexas (las cuales habrían necesitado de soportes para mantenerse erguidas) y en algunos casos son tetrápodas, es decir con patas que les aseguraban estabilidad (Gastaldi 2010). Las características de las pastas (porosas y de buen comportamiento térmico) y sus propiedades formales (formas globulares o subglobulares, sin puntos de inflexión marcados en el cuerpo) sugieren que eran recipientes aptos para ser sometidos al fuego (Gastaldi 2010). Presentan una altura que oscila entre los $240 \mathrm{~mm}$ y los $569 \mathrm{~mm}$, y diámetros máximos de cuerpo de entre $120 \mathrm{~mm}$ y $626 \mathrm{~mm}$; sus volúmenes también son variados, oscilando entre los 4,89 y los 101,07 litros, con una media de 62,78 litros. Las últimos cifras las colocan en el conjunto de recipientes con mayores capacidades de contención (tras las destinadas al almacenamiento), así como entre las de aparición más frecuente en los sitios (Gastaldi 2010).

Los patrones de carbonización y oxidación ${ }^{3}$ que existen sobre la superficie externa de estas vasijas fueron analizados por Gastaldi (2010), a partir de lo cual propuso tres

3 En algunos casos, se registra la presencia de zonas cubiertas con carbonizaciones y hollín, que cubren la totalidad de la superficie exterior de las piezas y suponen que los recipientes se encontraban un tanto alejados de la 


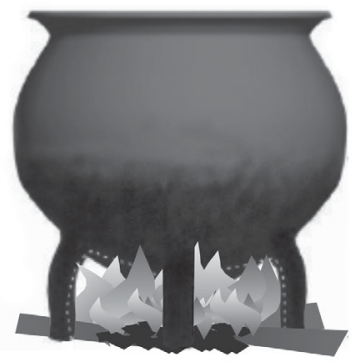

a
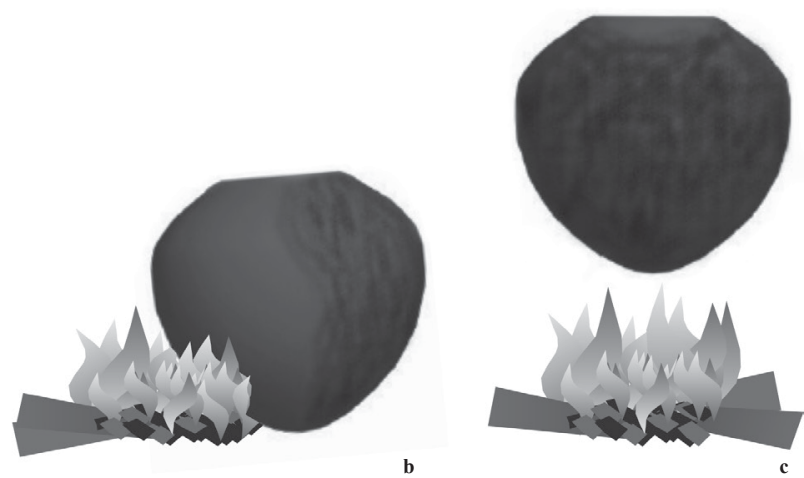

Figura 4: Formas de colocación de vasijas sobre el fuego: a) sobre el fuego, con contacto directo; b) sobre el fuego, de costado, con contacto directo; c) sobre el fuego, sin contacto directo.

(Basado en Gastaldi 2010).

formas en las que podrían haber sido colocadas sobre el fuego. En el caso de las vasijas tetrápodas (cuyas patas presentan una altura de $150 \mathrm{~mm}$ ), debieron ser colocadas sobre el fuego, en contacto directo con el mismo; el espacio de separación entre las patas sugiere que se habría tratado de fuegos pequeños (Figura 4a). En aquellas vasijas que no poseen patas, se registraron dos formas de colocación sobre el fuego. En el primer caso, se trata de una vasija que presenta un depósito de hollín restringido en uno de sus lados, por lo que debió ser colocada directamente sobre el fuego, de costado y ligeramente inclinada (quizá al rescoldo) (Figura 4b). Sobre un residuo carbonizado en el interior de esta vasija se realizó un análisis isotópico: su valor de $\delta^{13} \mathrm{C}(-12,2)$ fue consistente con el valor arrojado por maíz recuperado en el mismo sitio $(-12,1)$, lo que sugiere que este residuo se habría compuesto, al menos, de maíz y que se habría vinculado con este tipo de colocación sobre el fuego (Pazzarelli 2011). En el segundo caso, se trata de vasijas que presentan extensos depósito de hollín en toda la superficie, por lo que debieron ser colocadas por encima del fuego, sin contacto directo con el mismo (Figura 4c). En cinco residuos carbonizados del interior de vasijas de este tipo se recuperaron gránulos de almidón que podrían ser adscritos a Zea mays y que poseen muchos daños sobre su estructura (los cuales pueden corresponder a los procesos de cocción o bien a los procesamientos previos a la misma, como la molienda; Babot 2003, 2004); en dos de estos residuos se recuperaron los gránulos afines a tubérculos y raíces mencionados en el apartado anterior (lo cual permitió sugerir la cocción combinada de recursos).

Los estados en los que pudo haber ingresado el maíz en las ollas pudieron ser varios (tal como sugiere la culinaria actual), aunque los daños vinculados a la molienda hallados en los gránulos de uno de los residuos carbonizados y la ya mencionada alta frecuencia en el uso de molinos pueden sugerir que las harinas finas, con grandes capacidades de espesamiento, fueran un ingrediente usual. Es decir, además de su posible introducción como choclo o como grano fresco, también pudo ser hervido como mazorca seca, granos secos, granos partidos o como harinas de distinto tipo. Estos

fuente de calor, es decir, no tuvieron contacto directo con el fuego. En otros casos, existen fuertes oxidaciones, sin depósitos de hollín, lo que indica un contacto directo con el fuego. Estas huellas se combinan de diferentes maneras en los recipientes y permiten sugerir las diferentes formas en las que eran colocadas sobre el fuego (Gastaldi 2010). 
diferentes estados permiten pensar en los posibles controles culinarios realizados para lograr distintas texturas o densidades en las preparaciones hervidas. En este sentido, las capacidades de espesamiento de los granos partidos, de las harinas gruesas y de las harinas finas (de posible circulación frecuente) son diferentes (Babot 2004) y constituyen propiedades culinarias que suelen ser conocidas y manipuladas por los cocineros (Pazzarelli 2012a). Pero además, en el caso de Ambato tenemos que estos grados de espesamiento y viscosidad propios de los distintos estados del maíz pudieron combinarse con las propiedades de los tubérculos, ampliando las posibilidades culinarias.

A estas texturas debemos sumar los agregados de carnes y grasas. Un análisis de los índices de fragmentación de los conjuntos óseos de todos los sitios de Ambato permitió sugerir que el método usual de cocción habría sido el hervido, ya que este tipo de fracturas pudo vincularse a la necesidad de introducir los elementos óseos en las vasijas (Dantas 2010). Además, la relación entre esta fragmentación, la abundancia de huellas de procesamiento y la alta proporción de partes del esqueleto con gran cantidad de médula, sugiere que la grasa (en forma de grasa, médula, sesos y grasa ósea) habría sido de consumo frecuente en la dieta cotidiana (Dantas 2010), colaborando en los procesos de espesamiento en los que también se involucraban los distintos tipos de almidones y ampliando, al mismo tiempo, las posibilidades culinarias gracias a la combinación de recursos a partir de un mismo método de cocción (el hervido).

En suma, harinas finas de maíz, combinación de almidones e introducción de carnes y grasas en distintos estados habrían constituido los posibles criterios de definición de los preparados hervidos en Ambato. A ello se sumaban las diferentes formas de colocación sobre el fuego de las vasijas, que permitían preparados de distinto volumen y, posiblemente, con diferentes grados de concentración y espesamiento. Además, esto suponía un manejo de los aportes calóricos de los fogones, es decir de cocciones controladas que permitieran alcanzar las texturas deseadas. Todos estos procesos eran conducidos en el interior de las habitaciones o cocinas.

Respecto de otros tipos de cocción ${ }^{4}$, podemos citar la posibilidad del tostado, que requiere someter los alimentos al calor del fuego, mediado por algún elemento (como un fragmento de vasija, por ejemplo). En una pequeña mano de molino se identificaron granos de almidón con características similares a las que desarrolla Zea mays, que registraban daños posiblemente vinculados al tostado (Babot 2003, 2004). Algunos rastros similares se hallaron en gránulos que se encontraban en los residuos carbonizados de vasijas subglobulares, ya referidos. En ambos casos, sin embargo, se trata de indicios que deberían ser confirmados con nuevos análisis y aún deben ser considerados como hipótesis.

Dos notas podemos agregar respecto de las cocciones. Por un lado, que las vasijas mencionadas, lejos de haberse vinculado con usos exclusivos, podrían haberse

4 También existen evidencias de restos de maíces carbonizados hallados en los montículos-basureros de varios sitios (producto de desechos o bien de prácticas rituales vinculadas con estos espacios) y mazorcas carbonizadas que se encontraban vinculadas con el entierro de un camélido neonato. El registro de este tipo de transformación por exposición directa al fuego todavía es escaso y no podemos generalizar sobre sus implicaciones en la vida social; no obstante, es probable que sean el resultado de prácticas vinculadas a una (anti)culinaria que permitía establecer relaciones de comensalidad con agentes no humanos, como sucede actualmente con algunas comidas durante los pagos a la tierra en agosto, como argumentamos en otro trabajo (Pazzarelli 2012a). 
implicado en distintos eventos de cocción, de diferente intensidad y que hubieran requerido de un manejo diferencial de los aportes calóricos de los fogones. De hecho, aquellas utilizadas para hervir podrían haber sido utilizadas también para tostar en determinadas ocasiones. Por otro lado, que a pesar de estas posibles diferencias de manejos culinarios, todas las cocciones se habrían desarrollado en el interior de los recintos cerrados y techados, ya que el registro de estructuras de combustión se encuentra restringido a ellos. Es decir, mientras las «comidas húmedas» (sopas, guisos, preparaciones semilíquidas) podrían haber circulado por distintos lugares al ser consumidas, las etapas de transformación y cocción se vinculaban siempre con los espacios más cerrados (y menos visibles) de los sitios, a diferencia de otras técnicas culinarias que tenían lugar en espacios menos restringidos.

\subsection{Fermentaciones}

Los procesos de fermentación que incluyen al maíz son comunes en los Andes por constituir una de las transformaciones fundamentales a la hora de producir chicha. En Ambato, este proceso se relacionó, principalmente, con el mismo tipo de recipientes descrito para el almacenamiento: grandes vasijas, sin huellas de exposición al fuego y distribuidas, sobre todo, en las galerías (Figura 3). Dos líneas de evidencia permiten argumentar esta vinculación. En primer lugar, muchas de estas vasijas poseen un tipo particular de erosión en su superficie interior, caracterizada por una reducción en el espesor de la matriz arcillosa y por presentar antiplásticos sobresalientes, a manera de islas. A través de estudios experimentales, fue posible comprobar que este tipo de huellas se producen como consecuencia de las transformaciones tribo-químicas que supone la fermentación, lo cual permitió plantear la hipótesis de que las huellas registradas en las vasijas arqueológicas se vinculan con los mismos procesos (Gastaldi 2010; Pazzarelli 2012a) .

La segunda línea de evidencia la constituye el análisis de microfósiles y de sustancias adheridas. Por un lado, se han identificado gránulos de almidón afines a Zea mays sobre la superficie interna y externa de vasijas con ese tipo de erosión y, asimismo, sobre un residuo carbonizado adherido a una de ellas. En el último caso, los granos presentan importantes daños en su estructura (los cuales se reproducen en otros no identificados) que podrían vincularse a los diferentes e intensivos procesos de cocción que implica la producción de chicha o incluso a alteraciones producidas durante el proceso de fermentación (aunque la distinción entre ambas todavía no haya sido lo suficientemente estudiada; ver Babot 2004). Por otro lado, en el mismo

5 Estos patrones se identificaron también en otras vasijas, similares a las que describimos en el apartado dedicado a las cocciones, y que poseían huellas de carbonización en su exterior. Como la fermentación no requiere de exposición al fuego, es posible que estos recipientes fueran utilizados de forma secuencial, es decir, durante la primera parte de sus vidas como vasijas para fermentar y luego como ollas de cocina (Gastaldi 2010). Algo similar señalan distintos trabajos etnográficos: aquellos recipientes utilizados para la fermentación no son utilizados para otra tarea, con objeto de no alterar propiedades tales como la retención de fermentos en las superficies porosas de su interior que ayudan a acelerar nuevas fermentaciones (Cutler y Cárdenas 1985). De todas maneras, las grandes vasijas ubicadas en las galerías, sin rastros de hollín y con evidencias de erosión, constituyen, hasta el momento, el registro más directamente vinculado con la fermentación de bebidas a gran escala. 
residuo se realizó un análisis cromatográfico para la identificación de ácidos grasos que evidenció la presencia mayoritaria de ácidos (insaturados y de cadena larga) que pueden ser vinculados a recursos vegetales (argumento que podría ser reforzado por la presencia de granos de almidón) y de otros ácidos (ramificados de número impar) característicos de las grasas de rumiantes, como los camélidos (Pazzarelli 2011). Si bien en ninguno de los casos es posible realizar una adscripción definitiva a recursos particulares, el último conjunto de resultados permite sugerir algunas prácticas específicas vinculadas a estos recipientes: el almacenamiento de carnes (frescas y/o desecadas) y/o huesos, en momentos posteriores a su uso para la fermentación; o bien la introducción de carne como un ingrediente más del proceso, como lo sugiere la receta actual de la «chicha charque» (Paredes Candia 1990). Estas posibilidades deben seguir siendo exploradas, pero resultan interesantes para comenzar a formular hipótesis sobre estos posibles entramados de transformaciones donde, usualmente, las carnes de camélidos no se incluyen; y en el caso de que la chicha hubiera incorporado recursos cárnicos, esta combinación con el maíz debería sumarse a aquellas descritas en el apartado dedicado a las mezclas.

Por último mencionaremos el caso de otra vasija, hallada en la galería de un sitio de gran complejidad espacial («Piedras Blancas»), que presenta similitudes morfológicas con los puñus: recipientes cerámicos actuales, de borde muy evertido y cuello pequeño, que son especialmente manufacturados para fermentar chicha (Cutler y Cárdenas 1985). En su superficie interior también se registra la erosión característica de estos procesos y los análisis cromatográficos para la identificación de ácidos grasos también señalan una posible tendencia hacia recursos de origen vegetal (Pazzarelli 2011).

En virtud de constituirse en parte de una de las secuencias de transformación culinaria más compleja del mundo andino, la fermentación permite terminar de poner en relación, al menos en esta primera mirada, a los distintos tipos de objetos y transformaciones registrados en Ambato. Pues en la producción de chicha se involucra una gran diversidad de recipientes cerámicos, utilizados en distintas instancias (hervidos, decantados, mezclas, diluciones), tal y como lo atestiguan las miradas etnográficas sobre el tema (Cutler y Cárdenas 1985; Randall 1993). Estos procesos que ocurren en vasijas son habilitados por la presencia de harinas (que luego son mezcladas y hervidas) que se producen mediante el uso de piedras de moler. El proceso de producción de chicha, entonces, articulado en el marco de tres transformaciones claves (molienda, hervido y fermentación) ponía en relación a los distintos objetos que mencionamos y nos señala la circulación del maíz por diferentes clases de transformaciones, en un recorrido que va desde las galerías (en donde se encontraba el maíz almacenado y se molía) a los recintos (en donde se hervía) para salir nuevamente a los espacios semiabiertos (donde terminaba fermentando).

\section{Discusión: trayectorias, transformaciones, especificaciones}

Como anunciamos, nuestra tesis aquí es que en Ambato las trayectorias y transformaciones descritas operaban como un proceso de «especificación culinaria» que alteraba 
el aparente carácter único del maíz para socializarlo de diferentes maneras, como otrosmaices que a su vez servían para propiciar distintas relaciones de comensalidad. Repasemos estas trayectorias para otorgar mayor densidad explicativa a esta afirmación.

En primer lugar, el procesamiento y consumo de choclos frescos parece haber implicado la ejecución de técnicas (desgranado/rallado) y el uso de objetos (cuchillos de filita) que no volvían a vincularse al maíz en su estado deshidratado: tal vez eran exclusivas de los momentos posteriores a la cosecha. De la misma manera, habrían propiciado la generación de texturas y sabores que, de contar sólo con una cosecha anual, no podrían haber sido reproducidos en otros períodos del año; una suerte de tratamiento culinario diferencial que también ha sido referido en algunas etnografías (Gose 2001) ${ }^{6}$.

La deshidratación, en cambio, constituía una técnica que ubicaba al maíz en el largo término, extrayéndolo del plano de contingencia signado por la podredumbre que suponía su conservación en estado fresco. Una vez secos, los granos o las mazorcas podían ser almacenados en grandes vasijas colocadas, en su mayoría, en las galerías de los sitios. Esta ubicación de las reservas (secas y duras) en un espacio semiabierto de alta visibilidad y accesibilidad para los habitantes de la misma unidad doméstica quizá expresaba materialmente el estado de potencialidad (o «vitalidad», según algunos autores; van den Berg 1989; Allen 2002) y versatilidad de lo almacenado: es decir, la cantidad de caminos posibles que los granos podían transitar a partir de ese momento, gracias a su estado de sequedad.

Dos transformaciones eran las que ayudaban a extraer a los granos del largo término y a ubicarlos en el plano de la contingencia cotidiana: el (posible) tostado y la molienda. Sobre la primera, todavía no existe demasiada evidencia que permita contextualizarla adecuadamente, pero hemos sugerido la posibilidad de que se vinculara a la producción de harinas tostadas. Respecto de la segunda, suponía la ruptura y/o pulverización del maíz seco y duro, con objeto de lograr granos partidos o harinas de distintas cualidades que serían consumidas directamente o incluidas en otras preparaciones. Sugerimos que parte de esta producción podría haberse almacenado (¿las harinas de maíz tostado?), aunque en algunos casos esto hubiera supuesto procesos de mezcla (para lograr patay o bolanchao). El resto de los productos (harinas sin tostar) deberían haber sido cocidos (hervidos) y consumidos en los momentos casi inmediatamente posteriores a su producción. En este sentido, la molienda cotidiana (y el tostado, probablemente) ocupaba espacios similares a los del almacenamiento (es decir, galerías y lugares semiabiertos) y permitía actualizar la disponibilidad de un recurso deshidratado, al tiempo que habilitaba diferentes tipos de preparaciones, «secas» y «húmedas», con distintas posibilidades de conservación. En otras palabras, la molienda opera como un primer articulador entre los granos deshidratados y las harinas; esos nuevos otros-maices; en este sentido, podría considerarse una «operación estratégica» para el consumo del mismo (sensu Lemonnier 1992).

En el primero de los casos («comidas secas», como patay), las transformaciones podrían haber ocurrido en los espacios semiabiertos de las galerías o cerca de los vanos de acceso, donde es probable que adquirieran un carácter más visible vinculado

6 Gose (2001: 200) refiere el manejo culinario de los choclos frescos que se realiza en Huaquirca (Perú), donde se los considera como «recién nacidos» que deben ser tratados con técnicas culinarias (o más bien «técnicas sacrificiales») específicas. 
a las mencionadas posibilidades de transporte y circulación de este tipo de comidas, cuyas ingestas podían suponer vínculos con comensales diferentes a los de la misma unidad doméstica, tal como sucede actualmente (Weismantel 1994; Vokral 1991). Sus texturas finas quizá caracterizaron dichos eventos, como lo hacen actualmente al espesar infusiones, al empolvar las manos cuando se las consume directamente o al empastar las gargantas de los comensales (Spedding 1993; Gose 2001). En el segundo de los casos («comidas húmedas»), las harinas habrían ingresado hacia el espacio cerrado de las habitaciones, donde se ubicaban los fogones que permitían colocar las ollas a hervir, y donde seguramente se mezclaron con otros recursos, de origen vegetal y animal. Estas transformaciones eran menos visibles que las anteriores, más encerradas, y quizá se vincularan al carácter específico de las relaciones de comensalidad implicadas en su ingesta: consumos realizados tal vez en el interior de los recintos (o al menos de los sitios $)^{7}$, que suponían instancias regulares y diarias de agregación de los miembros de la unidad doméstica para compartir comidas semilíquidas y espesas (guisos o sopas), probablemente calientes, que debían ser contenidas en platos y consumidas con cucharas o directamente bebiéndolas. Todas estas preparaciones re-colocaban a las harinas/granos en un espacio de contingencia aún más ajustado que aquél que propiciaba la molienda, pues este tipo de comidas deberían haber sido consumidas (y preparadas) diariamente: de otra manera, se pudrían. Al mismo tiempo, no todas ellas podrían haber abandonado los recintos o los sitios para ser consumidas en otros espacios o, de haberlo hecho, su circulación no habría sido tan extendida como aquella que podían realizar las «comidas secas» y de mayores posibilidades de conservación. En este sentido, el hervido producía una clase de comidas (y un otro-maíz) que exigía un consumo diario y probablemente la concurrencia de todos los comensales en el mismo espacio en donde se lo había cocinado. Existe un caso, no obstante, en el que los productos del hervido podrían haber sido extraídos de los recintos y vueltos a re-transformar: la producción de chicha.

Los procesos de producción de chicha, como ya señalamos, son complejos y requieren de distintas instancias de transformación. En Ambato, el registro de vasijas destinadas a procesos de fermentación las ubica, sobre todo, en las galerías: es decir, si volvemos a atender a las secuencias de transformaciones en los sitios, tenemos que las harinas de maíz eran producidas (molidas) en espacios semiabiertos, luego ingresaban en las ollas para ser hervidas dentro de los recintos y finalmente volvían a salir hacia las galerías para concluir el proceso con la última etapa de transformación (la fermentación). Allí adquirían ese estado líquido (aunque algo espeso) y algo gaseoso (con burbujas) que caracteriza a esta bebida, junto con el grado alcohólico deseado. Entonces, mientras las comidas cotidianas abandonaban las ollas ya listas, las fracciones hervidas para la chicha debían continuar su transformación en el espacio de las galerías y en vasijas muy similares a aquellas que habían almacenado los granos por primera vez. Quizá esta homologación entre los recipientes permita ubicar, al menos parcialmente, a la chicha (que fermentaba en vasijas de forma «A») y a los granos secos (almacenados

\footnotetext{
7 Un análisis de las concentraciones de fosfatos y de materia orgánica en una de las habitaciones del sitio Piedras Blancas (Recinto F), demostró que las actividades cotidianas (entre las que se cuentan a las relaciones de comensalidad) se habrían desarrollado alrededor de los fogones presentes en el interior del recinto (Pazzarelli y Marconetto 2010; Pazzarelli 2012a).
} 
en los mismos tipos de recipientes) dentro de una misma categoría de sustancias con una alta potencialidad y versatilidad (¿y «vitalidad»?; van den Berg 1989; Allen 2002). Nuevamente, es probable que este carácter «visible» que adoptaba la fermentación se vinculara a las comensalidades colectivas que habilitaba una bebida de este tipo, que quizá implicaba la reunión de personas en los patios o su transporte a sitios o espacios destinados a eventos de agregación social (como los señalados más arriba, en la caracterización de los asentamientos de la cultura de La Aguada en Ambato).

En todos los casos mencionados arriba podemos observar que, al igual que en muchas otras regiones de los Andes, en Ambato el maíz se constituía en objeto de una diversidad de transformaciones, mezclas y consumos. Existía una manipulación y gestión de las transformaciones culinarias que se relacionaba con dinámicas domésticas, circulaciones y un uso diferencial del espacio que parece haberse vinculado y haber coadyuvado a la habilitación de distintas relaciones de comensalidad. El maíz se introducía de forma paulatina en los sitios residenciales, recorría diferentes espacios y en cada uno de ellos se vinculaba con objetos y técnicas particulares (algunas de ellas probablemente de carácter estacional), que alteraban su estado y le proporcionaban un nuevo «cuerpo» (duro, entero y seco; seco, blando y triturado; semilíquido, espeso y cremoso; burbujeante y alcohólico) y lo re-colocaban en nuevos planos de contingencia (Pazzarelli 2012a, 2012b). Esto nos permite comprender, en otras palabras, el modo en que el maíz se involucraba en una serie de relaciones múltiples (Laguens y Pazzarelli 2011) que terminaban por transformarlo en algo nuevo. La lógica de esas relaciones, recurrentes y estabilizadas a lo largo del tiempo, definía no sólo los modos en que el maíz iba a transformarse, sino también los espacios que cada uno de sus nuevos «cuerpos» iba a ocupar: los interiores eran el lugar del que podríamos llamar guiso-maíz, mientras que la galería y los vanos de acceso se encontraban poblados de harina-maíz o chichamaíz. Cada uno de estos nuevos cuerpos poseía conexiones parciales con las redes de relaciones que constituían a los otros (y tal vez la molienda, como una «operación estratégica», constituyó un punto crucial de estas conexiones), pero finalmente cada uno poseía también un camino específico que lo conducía a un destino particular (Laguens y Pazzarelli 2011). Es en este sentido que sería difícil argumentar que el maíz almacenado en vasijas era el «mismo» que el de los guisos que se consumían diariamente o el de la chicha que se bebía en eventos colectivos; era, más bien, un otro-maíz.

De este modo, antes que sostener que las transformaciones culinarias eran mediaciones para «aprovechar» recursos alimenticios, aquí afirmamos que deberían ser consideradas como un conjunto de técnicas específicas que permiten «producir» esos recursos y sustancias que aparentemente sólo se están «aprovechando» o «transformando». Antes de la ejecución de estas técnicas, las posibilidades de transformación del maíz no existen sino como una posibilidad (de tantas). Sólo las técnicas culinarias permiten actualizar esas posibilidades y propiciar un proceso que, al mismo tiempo que transforma un recurso, lo produce como uno nuevo: lo «especifican» y le otorgan un nuevo «cuerpo». En otras palabras, propician una operación de distinción en la que un maíz ya no es igual a otro, aunque ambos provengan de la misma planta ${ }^{8}$.

8 En este sentido, advertimos sobre las dificultades de la reificación de las identificaciones arqueobotánicas: ¿podríamos considerar que el maíz identificado en una olla es el «mismo» que el recuperado en una terraza de cultivo? (Pazzarelli 2012a). 
En el caso de Ambato, la indagación sobre estas transformaciones permite lograr una mejor comprensión de la organización lógica de las actividades domésticas, en donde cada transformación y cada producto contaba con un lugar dentro de los esquemas que se reproducían diariamente, unos esquemas que eran actualizados de la misma manera en toda clase de sitios, de mayor o menor complejidad, en virtud, podríamos pensar, de la relevancia que poseían para la reproducción de las relaciones de comensalidad que daban existencia y cohesión a los grupos. En todo caso (y esto debe seguir siendo explorado en la línea culinaria que propone este trabajo), aquellos sitios de gran envergadura que contaban con un mayor manejo de volúmenes de recursos (Laguens $2006,2007)$ podrían haber logrado amplificar sus posibilidades culinarias para auspiciar eventos de comensalidad colectiva en escalas mayores, pero siempre inscritas en el marco de las mismas lógicas culinarias (Laguens et al. 2007; Pazzarelli 2012a).

\section{Conclusiones}

Este trabajo ha tenido la intención de centrar el foco sobre algunos aspectos vinculados con las técnicas y transformaciones culinarias que tenían lugar en la vida cotidiana de Ambato. La información y el análisis del registro arqueológico se han puesto en relación con algunas discusiones ligadas a la organización de las prácticas domésticas en la región, con el lugar que la cocina ocupaba en la definición de las mismas y con los modos en que podrían haberse propiciado y habilitado diferentes relaciones sociales de comensalidad. En este sentido, y apelando a la inspiración de distintas herramientas teórico-analíticas (Viveiros de Castro 1986; Weismantel 1998; Vilaça 2002), hemos argumentado que los procesos de transformación lograban la socialización del maíz gracias a una «especificación culinaria» que producía otros-maíces a través de la generación de nuevos «cuerpos» (con ciertas texturas, densidades, temperaturas y mezclas), que permitía entonces su inscripción en distintas relaciones de comensalidad. Atendiendo a las lógicas locales, materiales y espaciales de su transformación observamos trayectorias que en ocasiones se solapaban y en otras se esquivaban (Laguens y Pazzarelli 2011); son esas recurrencias y distinciones las que permiten alegar a favor del tipo de proceso mencionado: el maíz molido y hervido en una olla junto a grasa animal y tubérculos no sólo es una versión transformada de los granos que se obtuvieron en la cosecha; es un otro-maiz. En otras palabras: a través del análisis del registro material de Ambato, estas páginas constituyen un intento de repensar las transformaciones culinarias lejos de los modelos teleológicos que las consideran «medios» para el consumo; desde nuestro punto de vista, por el contrario, se encuentran dotadas de un profundo potencial generativo y productivo que, en este caso, permiten hacer del maíz un otro.

\section{Referencias bibliográficas}

ABercrombie, Thomas

2006 Caminos de la memoria y del poder. Etnografía e historia en una comunidad andi- 
na. La Paz: Instituto de Estudios Bolivianos - Institut Français d'Études Andines - ASDI-SAREC.

Allen, Catherine Wagner

2002 The Hold Life Has. Coca and Cultural Identity in an Andean Community. Washington: Smithsonian Institution Press.

Aschero, Carlos A.

1975 Ensayo para una clasificación morfológica de artefactos líticos aplicada a estudios tipológicos comparativos. Informe inédito. Buenos Aires: CONICET.

Assandri, Susana

2007 Procesos de complejización social y organización espacial en el Valle de Ambato, Catamarca. Tesis de maestría. La Rábida: Universidad Internacional de Andalucía. http://www.unia.es/components/com_booklibrary/ebooks/0029_Assandri.pdf, con acceso el 26/9/2012.

Assandri, Susana, Adela Ávila, Rodolfo Herrero y Sofía Juez

1991 «Introducción a la biogeografía y arqueología del Valle de Ambato (Provincia de Catamarca, Argentina)». Publicaciones del CIFFYH Arqueología 46:7-16.

BАвот, María del Pilar

2003 «Starch Grain Damage as an Indicator of Food Processing», en Phytolith and Starch Research in the Australian-Pacific-Asian Regions: The State of the Art, D. M. Hart y L A. Wallis, eds., pp. 69-81. Canberra: Pandanus Books for the Australian National University.

2004 Tecnología y utilización de artefactos de molienda en el Noroeste prehispánico. Tesis de Doctorado Inédita. Universidad Nacional de Tucumán. Argentina.

BRAY, Tamara

2003 «To Dine Splendidly. Imperial Pottery, Comensal Politics, and the Inca State», en The Archaeology and Politics of Food and Feasting in Early State and Empires, Tamara Bray, ed., pp. 93-142. Nueva York: Kluwer Academia - Plenum Publishers.

CAPparelli, Aylén

2008 «Caracterización cuantitativa de productos intermedios y residuos derivados de alimentos del algarrobo (Prosopis flexuosa y P. chilensis, Fabacaeae): aproximación experimental aplicada a restos arqueobotánicos desecados». Darwiniana 46 (2):175-201.

Contreras Hernández, Jesús

1985 Subsistencia, ritual y poder en los Andes. Barcelona: Mitre.

Cutler, H. y M. CÁRDENAS

1985 «Chicha, una cerveza indígena sudamericana», en La tecnología en el mundo andino, Heather Lechtmann y Ana M. Soldi, coords., pp. 247-259. México: Universidad Nacional Autónoma de México.

DANTAS, Mariana

2010 Arqueología de los animales y procesos de diferenciación social en el Valle de Ambato, Catamarca, Argentina. Tesis doctoral inédita. Universidad Nacional de Córdoba.

FERnÁNDEZ JuÁREZ, Gerardo

1997 Entre la repugnancia y la seducción. Ofrendas complejas en los Andes del Sur. Cuzco: Centro de Estudios Regionales Andinos «Bartolomé de las Casas». 
FigueroA, Germán

2010 Organización de la producción agrícola en contextos sociales no igualitarios: el caso del valle de Ambato, Catamarca, entre los siglos VII y XI d.C. Tesis doctoral inédita. Universidad Nacional de Córdoba.

FigueroA, Germán, Mariana Dantas y Andrés Laguens

2010 «Prácticas agropastoriles e innovaciones en la producción de plantas y animales en los Andes sur. El Valle de Ambato, Argentina, Primer Mileno d. C». International Journal of Southamerican Archaeology 7: 6-13.

GASTALDI, Marcos

2010 Cultura material, construcción de identidades y transformaciones sociales en el Valle de Ambato durante el primer milenio d.C. Tesis doctoral inédita. Universidad Nacional de la Plata.

2012 «El lugar de los objetos en la teoría estratigráfica de Edward C. Harris: reflexiones desde una habitación del Valle de Ambato, Argentina». Intersecciones en Antropología 13 (1): 89-101.

Goldstein, Paul S.

2003 «From Stew-Eaters to Maize-Drinkers. The Chicha Economy and the Tiwanaku Expansion», en The Archaeology and Politics of Food and Feasting in Early State and Empires, Tamara Bray, ed., pp. 143-172. Nueva York: Kluwer Academic Plenum Publishers.

GoRDILLO, Inés

2009 El Sitio ceremonial de la Rinconada: Organización socio espacial y religión en el Valle de Ambato (Catamarca, Argentina). BAR International Series. Oxford: Archaeopress.

Gose, Peter

2001 Aguas mortiferas y cerros hambrientos. Rito agrario y formación de clases en un pueblo andino. La Paz: Mama Huaco.

HASTORF, Christine y Sissel JohANNESSEN

1993 «Pre-Hispanic Political Change and the Role of Maize in the Central Andes of Peru». American Anthropologist 95 (1):115-138.

LAGUENS, Andrés

2006 «Campo y espacio social en el estudio arqueológico de la desigualdad», en Contra la tiranía tipológica en arqueología: una visión desde Suramérica, Cristóbal Gnecco y Carl H. Langebaek, eds., pp. 99-119. Bogotá: Ediciones Uniandes.

2007 «Contextos materiales de desigualdad social en el Valle de Ambato, Catamarca, Argentina, entre el S. VII y X d.C». Revista Española de Antropología Americana 37 (1): 27-49.

Laguens, Andrés, Mariana Dantas, Germán Figueroa, Marcos Gastaldi, Sofía Juez y Francisco PAZZARELLI

2007 «Vasijas+pucos con huesos+agua no son sólo sopa: la cerámica de uso doméstico en el siglo XI d.C. en el Valle de Ambato (Catamarca) y sus relaciones con otros entramados sociales y materiales». Pacarina. Revista de Arqueología y Etnografía número especial: 353-359. San Salvador de Jujuy.

Laguens, Andrés y Francisco Pazzarelli

2011 «¿Manufactura, uso y descarte? O acerca del entramado social de los objetos cerá- 
micos». Revista del Museo de Antropología 4: 113-126. Córdoba.

LEMMONIER, Pierre

1992 Elements for an Anthropology of Technology. Ann Arbor: Museum of Anthropology, University of Michigan.

LÉvi-STRauss, Claude

1968 Mitológicas 1. Lo crudo y lo cocido [1964]. México: Fondo de Cultura Económica.

MurRa, John

1973 «Rite and Crop in the Inca State», en Peoples and Cultures of South America, Daniel Gross, ed., pp. 377-389. Nueva York: Doubleday and Natural History Press.

Nielsen, Axel

2010 Celebrando con los antepasados. Buenos Aires: Mallku.

Ossio, Juan

1988 «Aspectos simbólicos de las comidas andinas». América Indígena 48 (3): 549-570.

Paredes CANDiA, Antonio

1990 La comida popular boliviana (apuntes y recetario), 2a ed. La Paz: Librería Editorial Popular.

PAZZARELli, Francisco

2010 «La importancia de hervir la sopa: mujeres y técnicas culinarias en los Andes». Antípoda 10: 157-181.

2011 «Una aproximación a la transformación de recursos en Piedras Blancas (Ambato, Catamarca, S X-XI DC) desde los análisis químicos». Relaciones de la Sociedad Argentina de Antropología 36: 331-336.

2012a Arqueología de la comida. Cultura material y prácticas de alimentación en Ambato (Catamarca, Argentina). Siglos V-XI. Tesis doctoral inédita. Universidad Nacional de Córdoba.

2012b «El estudio de las transformaciones culinarias en los Andes: entre estructuras y superficies», en Las manos en la masa: arqueologías, antropologías e historias de la alimentación en Suramérica, M. del Pilar Babot, María Marschoff y Francisco Pazzarelli, eds., pp. 693-713. Córdoba: Universidad Nacional de Córdoba-Museo de Antropología (Universidad Nacional de Córdoba) - Instituto Superior de Estudios Sociales (Universidad Nacional de Tucumán).

Pazzarelli, Francisco y Bernarda Marconetto

2010 «En torno al fuego: fogones y comensalidades en Ambato (Catamarca, Argentina)», en Jornadas de arqueología de la alimentación. Cultura material, prácticas y significados: Libro de resúmenes, M. del Pilar Babot, María Marschoff y Francisco Pazzarelli, eds., pp. 76-7. Córdoba: Museo de Antropología - ISES - IAM.

Pérez Gollán, José Antonio

1991 «La cultura de La Aguada vista desde el Valle de Ambato». Publicaciones del CIFFYH. Arqueología 46: 157-173.

1994 «El proceso de integración en el Valle de Ambato: complejidad social y sistemas simbólicos». Rumitacana 1 (1): 33-41.

RANDALL, Robert

1993 «Los dos vasos. Cosmovisión y política de la embriaguez desde el incanato hasta la colonia», en Borrachera y memoria. La experiencia de lo sagrado en los Andes, Thierry Saignes, comp., pp. 73-112. La Paz: Hisbol - Institut Français d'Études Andines. 
Spedding, Alison

1993 «Semiótica de la cocina paceña andina o 'porquerías que se hacen pasar por comida' (un bosquejo inicial)». Unitas 10: 51-60. La Paz.

VAN DEN BERG, Hans

1989 La tierra no da así nomás. Los ritos agrícolas en la religión de los aymara-cristianos. Amsterdam: CEDLA.

VILAÇA, Aparecida

2002 «Making Kin Out of Others in Amazonia». The Journal of the Royal Anthropological Institute 8 (2): 347-365.

Viveiros de CASTRo, Eduardo

1986 Araweté. Os deuses canibais. Río de Janeiro: Jorge Zahar Editor - ANPOCS.

VOKRAL, Edita

1991 Qoñi-Chiri. La organización de la cocina y estructuras simbólicas en el Altiplano de Perú. Quito: Abya-Yala - COTESU.

Weismantel, Mary

1994 Alimentación, género y pobreza en los andes ecuatorianos. Quito: Abya-Yala.

1998 «Viñachina: hacer guaguas en Zumbagua, Ecuador», en Gente de carne y hueso. Las tramas del parentesco en los Andes, Denise Arnold, comp., pp. 83-96. La Paz: ILCA - CIASE. 